\title{
Anesthetic Technique and Resuscitation of a 38 Weeks Pregnant Patient with Established Anaphylactic Reaction-A Mysterious Case Report
}

\author{
Debabrata Banik ${ }^{*}$, Joysree Roy², AKM Faizul Haque ${ }^{3}$, AKM \\ Habibullah ${ }^{3}$, Mehdi Hassan ${ }^{3}$ and Moumita ${ }^{4}$ \\ 1Professor, Department of Anesthesia, Analgesia and Intensive Care Medicine. BSMMU, \\ Bangladesh \\ 2Professor department of Gynecology and obstetrics, Green Life Medical College, \\ Bangladesh
}

${ }^{3}$ Associate Professor; Consultant, Department of Anesthesia, Analgesia and Intensive Care Medicine, Bangabandhu Sheikh Mujib Medical University, Bangladesh

${ }^{4}$ Registrar, department of Gynecology and obstetrics, Green Life Medical College, Bangladesh

*Corresponding author: Debabrata Banik, FCPS MD, Professor, Department of Anesthesia, Analgesia and Intensive Care Medicine. BSMMU, Bangladesh,Email: banik85@gmail.com

\section{Abstract}

Introduction: Anaphylaxis reactions in pre-anesthetic period of 38 week of pregnant women are challenging for the anaesthesiologist who is dealing with anesthesia for urgent LSCS and resuscitation for both.. Anaphylaxis in pregnancy is uncommon condition with a prevalence of 2.7 cases/1 00000 deliveries.1-4 Aims of this case report is to realize the outcome of pregnant women with pre anesthetic anaphylaxis and find out the most effective anesthetic technique and management of pregnant patient.

Case: A 28 years old pregnant women was admitted for elective caesarian section and prophylactic cefuroxime sodium 1.5 gm given intravenously. The patient complained of restlessness, itching and respiratory distress which was improved by steroid. In the operation table all monitoring were attached, oxygen was given by nasal cannula, iv channel established and monitors were showing SPO2 99-100\% pulse 135-140 /min however, obstetrician didn't find fetal heart sound by fetoscope and stethoscope. Resuscitation and Anesthesia: Anaesthesiologist started immediate resuscitation considering anaphylactic reaction but improvement was very slow and consulted with obstetrician for communications with patient 


\section{Anaesthesia \& Critical Care Medicine Journal}

party regarding patient and fetal conditions with possible outcome, baby was delivered by LSCS with local infiltration anesthesia and supplement systemic analgesic.

Result: After delivery of the fetus all possible resuscitation efforts were taken but the baby didn't survive but the mother was improving. Mother was transferred to ICU discharged with full recovery after 2r hours.

Conclusion: It was may be slower onset presentation of anaphylactic reaction to cephalosporin antibiotic. It is a so rare case, proper management procedure and monitoring of mother and fetus was very difficult. Anesthetic technique for LSCS of established anaphylactic shock's pregnant patient is very challenging but it was successfully managed by local infiltration anesthesia with systemic analgesic.

Keywords: Anaphylactic reaction; Cephalosporins; Pregnant patient; Local infiltration anesthesia

\section{Introduction}

Anaphylaxis is a life-threatening severe condition due to type I hypersensitivity reaction1-2. It is a rare condition develops Anaphylaxis in pregnancy. It is an estimated prevalence of 2.7 cases $/ 100000$ deliveries [14]. Antibiotics are the most common identifiable cause of peri operative anaphylaxis 5-6, according a new Cleveland Clinic study-a finding that contrasts with results from several European studies. It is very important that maternal anaphylaxis can lead to significant fetal morbidity and mortality if uterine perfusion and maternal oxygenation are compromised [4,5]. It is common practice to uses prophylactic antibiotic before any surgery and it is also practice in obstetric cases. Cephalosporins are among the most commonly-used antibiotics in the treatment of routine infections, prophylactic and their use is increasing over time [6-9]. During the recent years anaphylactic reactions during anesthesia have become a common problem and are known to have caused of deaths in approximately $2 \%$ of the effected cases $[2,4,10,11]$. There is a significant risk of fetal/neonatal neurological damage or death even when the maternal outcome is favourable. The objective of this reporting of cases is to show that anaphylaxis reaction can occur with cephalosporin in pregnant patient with dangerous consequence. So Anesthetic management and resuscitation of 38 week of pregnant patient with established anaphylactic shock is extremely challenging for anesthesiologist.

\section{Reported Case}

A Obstetrician reported that a 28 years old women with 38 weeks pregnant (gravida 1, para 1) was admitted in a ward for elective caesarian section. On admission, the mother's vital signs were normal and general physical examination was unremarkable. A single intrauterine pregnancy with intact membranes was confirmed. The pregnancy had been uneventful with first and second trimester. S erology and the fetal sonograms were reported as normal. This patient was prepared for elective LSCS and according their protocol prophylactic antibiotic cefuroxime sodium $1.5 \mathrm{gm}$ was given intravenously. After given cefuroxime patient complained of restlessness, itching and some respiratory distress which was improved by administering steroid according to duty physician record. Her pulse, BP, respiratory rate and fetal heart rate was normal after and before cefuroxime injection according to ward duty physician's statement. The patient was send to Operation Theatre within one hour. All monitoring facility was attached, oxygen was given by nasal cannula, iv channel established and monitor was showing SPO2 $97-98 \%$ pulse $135-140$ /min. But obstetrician was charging fetal heart sound by fetoscope and stethoscope and did not confirm presence of fetal heart on operation table.

\section{Resuscitation and Anesthesia}

In the same time Anesthesiologist had taken the history and examined the patient as a schedule work. But Anesthesiologist founded that patient was drowsy, severe flushing of face, redness of whole body, no palpable peripheral pulse and non-recordable blood pressure in spite of all above monitoring finding. Started immediate resuscitation considering anaphylactic reaction inj, Adrenalin $100 \mu \mathrm{g}$ and Hydrocortisone $500 \mathrm{mg}$, IV fluid and wedge under buttock was given instantly and but improvement was very slow and consulted with obstetrician about urgent delivery of fetus for proper management of mother. Decision was made that baby 


\section{Anaesthesia \& Critical Care Medicine Journal}

was delivered by LSCS after proper explanation about mother and fetal condition with possible outcome to patient party. Anesthesiologist had no previous experience what type anesthesia will be provided in this situation. Instantly he decided with local infiltration anesthesia with supporting analgesia. Local infiltration anesthetic block was done by anesthesiologist with lignocaine $1 \%$ and bupivacaine $0.25 \%$ average $20 \mathrm{ml}$ and supported by oxygen initially then oxygen and nitrous oxide with spontaneous ventilation. After delivery of fetus analgesia was maintained by $20 \mathrm{mg}$ ketamine, $25 \mathrm{ug}$ fentanyl with spontaneous respiration and ergometrine was given for uterine contraction. Patient's hemodynamic condition was dramatically improved after delivery of fetus and placenta. Patient was good recovery and transfer the patient in ICU for better monitoring with continued the infusion of adrenaline and intermittent steroid for 24 hour. This patient was discharged from hospital without any abnormality and care of cephalosporin antibiotic allergen in future.

\section{Fetal Outcome}

At birth the female new-born were pale, hypotonic and no heartbeat, without spontaneous breathing or reflexes. The new-born was intubated and all measure of resuscitation was done for more than one hour but failed to survive the fetus.

Follow-up: The mother's hospital course was uneventful and she was discharged home 4 days later. The baby, however, was not so fortunate.

\section{Discussion}

This case represents a life-threatening morbidity and mortality to mother and her fetus respectively following the standard practice of providing prophylaxis antibiotic commonly used third generation Cephalosporin group. There is different studies report that Cephalosporins are among the most commonly-used antibiotics in the treatment of routine infections, and their use is increasing over time [6,7,12]. Anaphylaxis is a severe, sometimes life-threatening, allergic reaction that occurs within minutes to several hours of exposure an allergy-causing substance. (Medically reviewed by Drugs.com. Last updated on Feb 25, 2019. Harvard Health Publications). Anaphylaxis also is called anaphylactic shock and so serious allergic reaction that is rapid in onset and might cause death $[1,2,4]$. In our set up there is no data about incidence and prevalence of anaphylaxis in normal and pregnant women. But there is different author's report that the lifetime prevalence of anaphylaxis in the general population is estimated at $0.05-2 \% \cdot 10-15$ Data regarding prevalence among pregnant women is limited with an estimated prevalence near or at the time of delivery reported as 2.7 cases of anaphylaxis per 100000 deliveries [4,5]. There was a analysis of a State-wide hospital discharge database for last 20 years that total of 19 anaphylaxis pregnant cases were identify. Antibiotic penicillin groups were anaphylactic triggering of 11 and cephalosporin groups were anaphylectic triggering of 5 pregnant patients [4]. In this case antibiotic was used for surgical prophylaxis. All previously reported cases of antibiotic anaphylaxis during pregnancy have occurred in hospitalised women even though this may represent publication bias $[5,6,9,10,13,14]$. The cases have been reported in association with surgical prophylaxis prior to caesarean delivery, during prophylaxis of neonatal GBS infection or during treatment of maternal pyrexia.

About diagnosis of anaphylaxis during pregnancy is not different from a non-pregnant patients and it is mainly depend on a careful clinical history and physical examination $[1,2,15]$. But in this case sign symptom of anaphylaxis was not typical in immediate period but a slow onset of shock symptom [7,11-21]. There was no recordable blood pressure and skin redness due to wide speed vasodilatation of this study patient. Most of the previcious case report hypotension is the common sign almost $100 \%$ [4-9,22]. So Proper diagnosis was late and continuous monitoring was absent in this cases. Cephalosporins can cause a range of hypersensitivity reactions, from mild, delayed-onset cutaneous reactions to life-threatening anaphylaxis in patients with immunoglobulin E (IgE)-mediated allergy [9-13,23]. Anaphylaxis reactions to cephalosporins may be divided into immediate reactions, which develop within 1 hour after administration and non-immediate reactions, which develop $>1$ hour after administration [6,11,24]. All previous case review focuses on allergic reactions during the peripartum period with the differential diagnosis and specific treatment options related to specific agent 4 . During pregnancy Immunoregulation and susceptibility to allergic reactions may change due to hormonal level. Drug treatment of established anaphylaxis of pregnant women is not different from non-pregnant women but different is outcome of fetus and fetus delivery $[4,9,11]$. Although fetal outcome is important, the mother is the primary focus of care [13]. In this case resuscitation was started by standard drug adrenaline $100 \mu \mathrm{g}$ intravenously with fluid and steroid in the operation table before induction of anesthesia. But improvement was very slow with above 


\section{Anaesthesia \& Critical Care Medicine Journal}

drug treatment. So urgent LSCS was immenent for the patient of 38 week pregnant with nonaudiable fetal heat sound, but it was very challenge for anesthesiologist to select the technique of anesthesia. There was an analysis of a State-wide hospital discharge database for last 20 years that total of 19 anaphylaxis pregnant cases were identified. Among them 13 was gestational age of 36 to 40 week one was 35 other were 23 to 32 weeks pregnancy. So most of the patient were needed emergency delivery of fetus [4-6].

There is controversies exist regarding the best timing and mode of delivery of the neonate following anaphylaxis during pregnancy [4]. But there is an opinion for better outcome for the mother and neonate when maternal resuscitation was inadequate, immediate caesarean delivery may provide. With proper explanation about patient and fetal condition with possible outcome to patient party, baby was delivered by LSCS with local infiltration anesthesia and supplement systemic analgesic. There was no definitive protocol for technique of anesthesia of a pregnant patient with anaphylactic shock. Any form of anesthesia is a high risk due to severe hemodynamic unstable pregnant patient. Most of the Anesthetic drugs may be responsible for hemodynamic instability and also have properties of allergen [1-3,25]. Neuromuscular blocking agents and induction angent are frequently involved during perioperative anaphylaxis at a range of 50-70\% according to different reports in Europe [1-2,11]. Whereas limited data are available in the United States. Anaphylaxis to local anesthetics is very uncommon and has decreased in frequency because of the decreasing use of the ester group of local anesthetics. So choice of local infiltration anesthesia was done and effectively managed with excellent outcome of mother but fetal outcome was grave. There are some indication and case report that LSCS is done under local infiltration anesthesia [26-29]. But most of cases are used local anesthetic infiltration, entonox inhalation and letter on fentanyle with spontaneous ventilation. In this case used analgesis dose of ketamine and 25 ug fentanyle after delivery of fetus. There was no effect on respiration and positive effect on hemodynamic. There are also reported local anesthesia for LSCS causes loss of pain sensation in selected areas only, with minimal disturbances of other systems, especially the cardiovascular and respiratory system with noted that incidence of complications after using local anesthesia for LSCS, including fetal demise, was significantly lower $[28,29]$. There is no evidence that Cesarean section under local anesthesia has an increased incidence of mortality than any other form of anesthesia [29]. In this case death fetus was delivered which was very disgusting both for surgical team and patient family. There was an analytic report of a State-wide hospital discharge database for last 20 years that total of 19 anaphylaxis pregnant were delivered. Among them 8 fetus had developed neurological damage of different degree, 7 fetus had good APGAR score and 4 fetus died before and after delivery [4,5,8,9,30,31]. There was also reported a case of improved anaphylaxis pregnant patient readmitted within 24 hour of discharged and delivered the fetus [4-5]. This fetus died within 72 hour of delivery. In this case, diagnosis and stabilization of the mother in the ward was probably insufficient to prevent fetal damage and the reported final outcome. So there is must be a hospital protocol for continuous vigilance, high skill knowledge and always a degree of suspicion are essential for prompt diagnosis and treatment. A national and international consensus may be essential for the management of anaphylaxis in pregnancy and also it is highly desirable for technique of anesthetic management.

\section{Conclusion}

Obstetrician, anesthesiologists, resident doctors and nursing staff shouldn't forget the possibility of any kind of allergy or anaphylaxis during administration of any suspicious drug specially in a pregnant patient. Anaphylaxis in pregnancy with or without immediate cardinal sign is an increased risk of fatality for both mother and fetus and more for fetus with hypoxic/ischemic encephalopathy or death. Proper anesthetic technique with high skill of resuscitation will prevent the grave outcome of pregnancy. Usually do not advocate the use of local anesthesia for all Cesarean sections; it can be safely used in high-risk patients like severe anaphylactic shock where sub-arachnoid block or general anesthesia can be associated with complications

Recommendation: Perform skin test when administers any suspected allergen specially in pregnant patient. So the development and implementation of a maternal anaphylaxis management protocol by a multidisciplinary team of obstetricians, neonatologists and anaesthesiologists is highly recommended.

Acknowledgment: We would like to thank all the members of the operation theatre, patient and patient's family. 


\section{Anaesthesia \& Critical Care Medicine Journal}

\section{References}

1. Pascale D, Claudie Mouton F, Charles W Emala (2009) Anaphylaxis and Anesthesia: Controversies and New Insights. Anesthesiology 111(5): 1141-1150.

2. Harper NJ, Dixon T, Dugue P, Edgar DM, Fay A, et al. (2009) Suspected anaphylactic reactions associated with anaesthesia. Anaesthesia 64(2): 199-211.

3. Lieberman P (2002) Anaphylactic reactions during surgical and medical procedures. J Allergy Clin Immunol 110(2): 64-69.

4. Mulla ZD, Ebrahim MS, Gonzalez JL (2010) Anaphylaxis in the obstetric patient: analysis of a statewide hospital discharge database. Ann Allergy Asthma Immunol 104(1): 55-59.

5. Chaudhuri K, Gonzales J, Jesurun CA, Ambat MT, Mandal Chaudhuri S (2008) Anaphylactic shock in pregnancy: a case study and review of the literature. Int J Obstet Anesth 17(4): 350-357.

6. Sengupta A, Kohli JK (2008) Antibiotic prophylaxis in cesarean section causing anaphylaxis and intrauterine fetal death. J Obstet Gynaecol Res 34(2): 252-254.

7. Adriaensens I, Vercauteren M, Soetens F, Janssen L, Leysen J, et al. (2013) Allergic reactions during labour analgesia and caesarean section anaesthesia. Int J Obstet Anesth 22(3): 231-242.

8. Berardi A, Rossi K, Cavalleri F, Simoni A, Aguzzoli L, et al. (2004) Maternal anaphylaxis and fetal brain damage after intrapartum chemoprophylaxis. J Perinat Med 32(4): 375-377.

9. Sleth JC, Lafforgue E, Cherici O, Nagy P (2009) Anaphylaxis in terminal pregnancy: two case studies and review of the literature. Ann Fr Anesth Reanim 28(9): 790-794.

10. Simons FE (2010) Anaphylaxis. J Allergy Clin Immunol 125(2): 161-181.

11. Lieberman P, Camargo CA, Bohlke K, Jick H, Miller RL, et al. (2006) Epidemiology of anaphylaxis: findings of the American College of Allergy, Asthma and Immunology Epidemiology of Anaphylaxis Working Group. Ann Allergy Asthma Immunol 97(5): 596-602.
12. Simons F, Estelle R, Ardusso L (2011) World Allergy Organization Guidelines for the Assessment and Management of Anaphylaxis. WAOJ 4(2): 13-37.

13. Alberto Berenguer, Alexandra Couto, Vera Brites, Rosario Fernandes (2013) Anaphylaxis in pregnancy: a rare cause of neonatal mortality.

14. Schatz M (2012) Recognition and management of allergic disease during pregnancy. In: Basow DS, (Eds.), Up-to-date. Waltham, MA.

15. McCaig LF, Hughes JM (1995) Trends in antimicrobial drug prescribing among office-based physicians in the United States 273(3): 214-219.

16. Norrby SR (1987) Side effects of cephalosporins. Drugs 34 (2): 105-120.

17. Kelkar PS, Li JT (2001) Cephalosporin allergy. N Engl J Med 345(11): 804-809.

18. Khan R, Anastasakis E, Kadir RA (2008) Anaphylactic reaction to ceftriaxone in labour. An emerging complication. J Obstet Gynaecol 28(7): 751-753.

19. Konno R, Nagase S (1995) Anaphylactic reaction to cefazolin in pregnancy. Asia-Oceania J Obstet Gynaecol 21(6): 577-579.

20. Dunn A, Blomquist J, Khouzami V (1999) Anaphylaxis in labor secondary to prophylaxis against group B Streptococcus: A case report. J Reprod Med 44(4): 381-384.

21. Jao M, Cheng P, Shaw S, Soong YK (2006) Anaphylaxis to cefazolin during labor secondary to prophylaxis for group B Streptococcus: a case report. J Reprod Med 51(8): 655-658.

22. Luciano R, Zuppa AA, Maragliao G, Gallini F, Tortorolo G (1997) Fetal encephalopathy after maternal anaphylaxis. Case Report Biol Neonat 71: 190-193.

23. Antonino Romano (2019) Cephalosporin allergy: Clinical manifestations and diagnosis Anaphylaxis. Harvard Health Publication. Medically reviewed by Drugs.

24. Gei AF, Pacheco LD, Vanhook JW, Hankins GD (2003) the use of a continuous infusion of epinephrine for anaphylactic shock during labor. Obstet Gynecol 102(6): 1332-1335. 


\section{Anaesthesia \& Critical Care Medicine Journal}

25. Chao CP, Zaleski CG, Patton AC (2006) Neonatal hypoxic-ischemic encephalopathy: multimodality imaging findings. Radiographics 26(S1): 159-172.

26. Patil S, Sinha P, Krishnan S (2007) Successful delivery in a morbidly obese patient after failed intubation and regional technique. Br J Anaesth 99(6): 919-920.

27. Nandgopal M (2001) Local anesthesia for caesarean section. Tech Reg Anesth Pain Manag 5(1): 30-35.

28. Ranney B, Stanage WF (1975) Advantages of local anesthesia for caesarean section. Obstet Gynecol 45(2): 163-167.
29. Bablesh Mahawar, Neha Baduni, Pooja Bansal (2014) Cesarean section under local anesthesia: A step forward or backward? J Anaesthesiol Clin Pharmacol 30(4): 578-579.

30. Romano A, Torres MJ, Namour F (2002) immediate hypersensitivity to cephalosporins. Allergy 57(72): 52.

31. Madaan A, Li JT (2004) Cephalosporin allergy. Immunol Allergy Clin North Am 24(3): 463-476. 